\title{
RADIO SUPERNOVAE AS DIRECT EVIDENCE OF STELLAR EVOLUTION IN REAL TIME
}

\author{
S.D. VAN DYK \\ Visiting scientist, UCLA, USA \\ vandyk@astro.ucla.edu \\ M.J. MONTES AND K.W. WEILER \\ Naval Research Lab, USA \\ mmontes@moon.nrl.navy.mil, kweiler@sne.nrl.navy.mil \\ R.A. SRAMEK \\ $N R A O / V L A, U S A$ \\ dsramek@zia.aoc.nrao.edu \\ AND \\ N. PANAGIA \\ $S T S C i / E S A$ \\ panagia@stsci.edu
}

The radio emission from supernovae provides a direct probe of a supernova's circumstellar environment, which presumably was established by mass-loss episodes in the late stages of the progenitor's presupernova evolution. The observed synchrotron emission is generated by the SN shock interacting with the relatively high-density circumstellar medium which has been fully ionized and heated by the initial UV/X-ray flash. The study of radio supernovae therefore provides many clues to and constraints on stellar evolution. We will present the recent results on several cases, including SN 1980K, whose recent abrupt decline provides us with a stringent constraint on the progenitor's initial mass; SN 1993J, for which the profile of the wind matter supports the picture of the progenitor's evolution in an interacting binary system; and SN 1979C, where a clear change in presupernova mass-loss rate occurred about $10^{4}$ years before explosion. Other examples, such as SNe $1994 \mathrm{I}$ and $1996 \mathrm{cb}$, will also be discussed. 